\title{
A Survey of Mathematics-Based Equivalent-Circuit and Electrochemical Battery Models for Hybrid and Electric Vehicle Simulation
}

\author{
Aden Seaman ${ }^{\mathrm{a}, *}$, Thanh-Son Dao ${ }^{\mathrm{b}}$, John McPhee ${ }^{\mathrm{a}}$ \\ ${ }^{a}$ Systems Design Engineering, University of Waterloo, Waterloo, Ontario, Canada. N2L 3G1 \\ ${ }^{b}$ Maplesoft, 615 Kumpf Drive, Waterloo, Ontario, Canada. N2V 1 K8
}

\begin{abstract}
In this paper, we survey two kinds of mathematics-based battery models intended for use in hybrid and electric vehicle simulation. The first is circuit-based, which is founded upon the electrical behaviour of the battery, and abstracts away the electrochemistry into equivalent electrical components. The second is chemistry-based, which is founded upon the electrochemical equations of the battery chemistry.
\end{abstract}

Keywords: battery, model, circuit, electrochemical, HEV

\section{Introduction}

Battery modelling is a challenging field that has been receiving a great amount of interest recently due to two main commercial drives: the desire for longer-lasting portable electronic devices [1-3], and the great push for hybrid and battery electric vehicles by the automotive industry $[4,5]$.

These two industries have different aims. The portable electronics industry is concerned with maximizing the operating life of a low-power electronic device that runs for a long period of time on a small inexpensive battery pack. The electric vehicle industry is concerned with maximizing the driving range and fuel economy of hybrid and electric vehicles using large battery packs in demanding applications that involve high power charge and discharge rates that push the batteries to their limits, while operating within a range that maximizes the expensive battery pack's service life.

In both of these areas, accurate and efficient battery modelling is vital to help maximize the performance of a device and its battery, and to inform the development of electronic and control systems. The overall performance and life of the device depends on the control system and electronics that interface with the battery, which need to be carefully tailored to the behaviour of the battery. Furthermore the size and configuration of the battery pack must be chosen to maximize the performance of the device while minimizing its cost.

\footnotetext{
*Corresponding Author. Tel: +1-519-888-4567, Fax: +1-519-746-4791

Email addresses: anseaman@uwaterloo.ca (Aden Seaman), tdao@maplesoft.com (Thanh-Son Dao), mcphee@uwaterloo.ca (John McPhee)

Preprint submitted to Power Sources 
In this paper we focus on the field of battery modelling for automotive electric vehicle simulation, with an aim to help engineers and scientists become familiar with the field of battery modelling and the different models and techniques that are commonly used. Because the field of battery modelling is so extensive, we limit ourselves to considering only the most common battery chemistries and modelling techniques.

We also limit ourselves to acausal, physics-based battery models. This means our batteries use voltage, current, and temperature as the physical quantities of interest, and that they can be written as a system of continuous-time equations. This is in contrast to causal, signal-based battery models that typically use discrete-time equations in an iterative solution, and power as the main physical quantity of interest.

The most commonly used batteries in electric vehicles today are the Nickel-MetalHydride (NiMH) and Lithium-Ion (Li-ion) chemistries [6]. In the past, Lead-Acid batteries $(\mathrm{PbA})$ were used, but these are falling out of favour to the higher energy and power densities of the Ni-MH and Li-ion chemistries. However many of the techniques developed for modelling these PbA batteries are applicable to more modern chemistries. In the future, Li-ion is a very promising chemistry that is light weight and has high energy and power densities; however more research needs to be done to drive down the cost of these batteries and to increase their safety [6] and performance, particularly at relatively high and low temperatures.

In Section 2 we survey battery models as they apply to various battery modelling tasks. Section 3 focuses on equivalent circuit models in particular, and surveys different modelling techniques and considerations. Section 4 surveys electrochemical battery modelling, and Section 5 briefly compares an equivalent circuit and electrochemical battery under pulse discharge, shows the results of a sensitivity analysis of the two battery models, and finally shows the results of an electric vehicle simulation. The paper is finished off by the conclusions, acknowledgements, references, and appendices.

\section{Battery Modelling}

The two most common techniques we encountered for modelling batteries in automotive applications are equivalent electric circuit and electrochemical modelling. Some models [7-9] combine elements of both chemistry and circuit-based modelling techniques.

Equivalent circuit modelling techniques abstract away the electrochemical nature of the battery and represent it solely as electrical components [1]. Sometimes these contain non-linear components like diodes that strive to better approximate the electrochemical nature of the battery. The structure of the model depends on the type of experimental method used to determine the parameters of the model - which is usually either electrochemical impedance spectroscopy or measuring pulse discharge behaviour - as well as the desired fidelity and goals of the modelling effort.

Electrochemical modelling techniques are all based on the highly non-linear equations that describe the electrochemical physics of the battery, and employ many different approximations to simplify the equations and the solutions thereof, depending on the level of fidelity one requires and the goals of modelling the battery.

Generally, the simpler the model, the faster it will simulate, but the lower its fidelity. This is an important trade-off that one must consider when choosing a battery model to suit one's application, particularly if real-time simulation is a requirement. 
Although a comprehensive comparison of different models would be challenging due to the wide variety of phenomena different models are good at capturing, comparing similar models is possible. Zhang and Chow [10] do a computational complexity versus modelling error analysis for a Thévenin resistor-capacitor network circuit model, varying the number of $\mathrm{RC}$ pairs. Increasing the number of $\mathrm{RC}$ pairs decreases the error and increases the computation time, and this paper shows how these quantities scale as a function of RC pair number.

For real-time control system applications, where high fidelity models are often not required, simple circuit based models are employed [11-14]. However for applications requiring higher fidelity such as vehicle performance, drive cycle simulations, battery ageing, and other computation-intensive simulations, a higher fidelity circuit-based or chemistry-based model can be used to increase the accuracy of the results. Thus, the choice of the right battery model to use depends on the fidelity one requires for one's application.

For on-line state of charge (SOC) estimation, battery models are usually fairly simple, as they are fused with an actual battery using a technique like an Extended Kalman Filter (EKF) [15-17], a fuzzy-logic system [18, 19], a least-squares regression model [20], or a sliding-mode observer model [21]. What is usually required in these applications is that the model be simple enough to run on an embedded controller while being accurate enough to model the battery's internal variables of interest, primarily the SOC [22].

For control system development, battery models can again be fairly simple. Being able to run in real-time and on embedded computers is an important requirement for control systems, so the battery models can not be too computationally expensive. The majority of these models are circuit-based, comprising either a simple resistor, or a Thévenin resistor-capacitor network [11-14].

When doing fuel economy and vehicle performance simulations using drive cycles, more accurate models are desired to fine-tune the performance of the vehicle and power management controller to see how they perform over a long period of time with successive charge and discharge cycles. Since these calculations are usually executed offline on workstations, the stringent requirements of real-time computation are absent, and one can afford to use a more computationally expensive model such as the Li-ion models of $[8,10,23-32]$, the NiMH models of $[7,9,23,33]$, or the lead-acid models of $[23,34,35]$. A good comparison of circuit-based models for Li-ion batteries is presented by $\mathrm{Hu}$ et al. in [36].

If one is analysing the response of the battery to transients, a model that is based on these measurements and takes the dynamic battery response into account is desired. The Thévenin models of $[13,23,24,26,33]$ and the electrochemical impedance spectroscopy (EIS) models of $[7,8,22,37-39]$ are good examples of these. For high frequency switching, a greater number of resistor-capacitor (RC) pairs are necessary to better approximate the short time behaviour of the battery [23]. Alternatively one can model the mostly inductive behaviour of a battery in the high-frequency region using resistor-inductor (RL) networks [40].

An important consideration for electric vehicle applications is the battery's dependence on temperature, especially in cold climates. Because the rate of chemical diffusion is slowed by low temperatures, this can have a serious impact on the current-delivering capabilities of the battery. Although high temperatures have the opposite effect, the downside is that the rate of detrimental electrode oxidization is increased, which short- 
ens the service life of the battery $[1,41]$.

Many battery models do not consider temperature variations. If this is a modelling requirement, then it is necessary to use models like those proposed in [7-9, 25-31, 34] that include the effects of temperature on the dynamic response and state-of-charge behaviour of the battery.

State of health $(\mathrm{SOH})$ is a more difficult quantity to measure. It represents the battery's gradual loss of maximum capacity and increase in internal resistance over a long period of time, which eventually results in the battery needing to be replaced. It is due to gradual chemical effects changing the structure of the battery, mainly electrode oxidization, active material degradation, and electrolyte decomposition [1, 42]. These are hastened by high operating temperature, overcharging, and deep discharging.

Experimentally, $\mathrm{SOH}$ is a time-consuming effect to measure, as it changes slowly over hundreds to thousands of battery charge-discharge cycles.

In battery models, the effects of $\mathrm{SOH}$ are incorporated or measured in different ways. Bhangu et al. [15] use a circuit model with an EKF to determine SOH by measuring the change in bulk capacitance. Prada et al. [7,8] use EIS and an electrochemical ageing model to measure degradation. Troltzsch et al. [42] use a multi-frequency EIS approach with battery model parameter identification to match battery ageing with changes in model parameters. Salkind et al. [19] use a fuzzy-logic system to determine SOH in many battery chemistries. Erdinc et al. [28] use a resistance element that depends on the cycle number. A detailed review of using EIS to measure $\mathrm{SOH}$ is presented in [43], a small table of SOH measurement methods is presented in [5], and Zhang and Lee review prognostics and health monitoring of Li-ion batteries in [44].

There is no single model that is capable of meeting all the requirements one may have. Thus, it is important to consider one's modelling requirements and select which battery model best matches those requirements.

If one is using an electric circuit model and has a particular battery one wants to model, it is necessary to perform experimental measurements to get data that are then converted into the parameters of the model. The model by Gao et al. [26] can use information from manufacturer's data sheets to help determine its parameters. Alternatively if one has a detailed chemistry model, one could use it to generate the necessary data.

In any case, one must identify the circuit model parameters from battery chargedischarge data. Many people who use Thévenin models identify the circuit parameters using the battery's time response to pulse discharges [24, 27, 31, 33]. By looking at the instantaneous voltage drop, the time constants of the charge depletion and recovery regions, and the current, one is able to identify the resistors, capacitors, and open circuit voltage for a particular SOC. Kim and Qiao [45] apply a least-squares method to this pulse-discharge technique. Hu et al. [46] apply an algebraic simplification to the non-linear battery equations to ease the identification of circuit components from pulse discharge data.

Other people use more complicated techniques that compare the battery model simulation to the actual battery response with an objective function. Hu et al. [36] use multi-swarm particle swarm optimization to match several different battery models to the same experimental data. Hu et al. [29,30] use a genetic algorithm and 1D and 2D spline interpolation to find the SOC and temperature dependence of circuit model component values for charge and discharge current directions. Troltzsch et al. [42] use a combination of genetic global optimization and Levenberg-Marquardt local optimization 
to find parameter values for an EIS circuit model.

Unlike the chemistry-based models, the circuit-based models are not able to adapt to changing an arbitrary physical parameter of the battery, for example the molality of the electrolyte or the surface area of the anode, since the circuit model parameters do not correspond directly to these physical entities. Since parameter identification techniques are used to determine the values of circuit model components, it is necessary to obtain experimental or simulated data in order to tailor the circuit model to a particular kind and size of battery. An exception to this is when using batteries that differ only in size, as the currents and capacities can be normalized and scaled between batteries [38]. Battery packs of different sizes, but using identical battery cells, can also be modelled by scaling the current and voltage appropriately.

The following subsections provide more detail on the different techniques in circuit and chemistry-based battery modelling.

\section{Equivalent Electrical Circuit Models}

Circuit-based models attempt to model the electrochemical physics of a battery using only electrical components. These component models can easily be incorporated into the system model of an electric vehicle, and are generally not computationally expensive. Some of them can also be used in circuit-simulation environments such as SPICE [35, $47,48]$.

The simplest circuit model is the resistance model, shown in Figure C.1, with terminal equations described by Equation 1. This uses an open circuit voltage $\mathrm{V}_{\mathrm{oc}}$ based on the battery's state of charge (SOC) and a resistor $\mathrm{R}$ to model the equivalent series resistance of the battery [35]. A load $\mathrm{Z}_{\mathrm{L}}$ is connected across the battery terminals with voltage $\mathrm{V}(\mathrm{t})$ and current $\mathrm{I}(\mathrm{t})$ measured at the load. To keep track of the SOC, current integration (also called Coulomb-counting) is used.

$$
V(t)=V_{o c}-R I(t)
$$

Current integration, described by Equation 2, is the simplest way of keeping track of a battery's SOC, which ranges from 0 (empty) to 1 (full). $C_{N}$ is the battery's rated charge storage capacity and $I(t)$ is the battery current. The current consumed by loss reactions can also be accounted for by subtracting it from the battery current.

$$
S O C=S O C\left(t_{0}\right)+\frac{1}{C_{N}} \int_{t_{0}}^{t} I(\tau) d \tau
$$

For simulations, Coulomb-counting is acceptable, but in practice it suffers from drift. Thus, in the field it is only accurate for short durations, or when the battery is frequently recharged to a known SOC. A comprehensive overview of a variety of SOC determination techniques can be found in $[44,49,50]$.

The first step in increasing the fidelity of the simple resistor model is to make the resistor and open-circuit voltage dependent on SOC and temperature $[25,51]$, or by incorporating non-linear components such as diodes to change the battery parameters under charge and discharge conditions [11, 16, 35]. 
Further increases in fidelity can be achieved by incorporating more circuit components into the model, such as a network of resistors and capacitors to model timedependent effects. These higher-fidelity models can be divided into two main groups: Thévenin models and impedance models. The Thévenin models usually comprise a series of resistor-capacitor pairs, and have their model parameters identified by using pulse charge/discharge techniques. The impedance models usually incorporate a constantphase Warburg element and have their model parameters identified using EIS.

\subsection{Thévenin Models}

As mentioned above, Thévenin models generally consist of a network of a series of parallel resistor-capacitor pairs. These models can capture the time-dependent effects of charge depletion and recovery.

Charge depletion is an effect seen when one first begins to discharge a battery [52]. Due to an initially high concentration of chemical products near the cathode and anode the initial battery voltage drop is subdued and gradually decreases as chemical products are consumed. Charge recovery is the opposite effect, where the battery seems to recharge itself after discharging has stopped, due to chemical products diffusing from within the body of the battery to the anode and cathode. These behaviours can be seen when pulse discharging a battery, as illustrated in Figure C.2, and a simple explanation for this is given in [1].

A simple model that can capture these effects is shown in Figure C.3, incorporating one or more parallel resistor-capacitor networks in series. Here $\mathrm{R}_{\mathrm{s}}$ is the series resistance that models the instantaneous change in voltage with respect to current, and the $\mathrm{R}_{i} \mathrm{C}_{i}$ parallel pairs, where $i \in\{1, . ., N\}$, model the exponentially decaying time-dependent variation of voltage with respect to current. The circuit component values can be found using pulse discharge analysis $[24,27,31,33,45,46]$, or parameter identification techniques $[29,30,36]$. Variations on this parallel-RC model are the most common circuit models encountered by the authors.

The fidelity of these Thévenin models can be improved by making the battery component values depend on various internal states of the battery. The most basic improvement is to incorporate a dependence on the battery's SOC [10, 23, 24, 27-32, 35, 45, 46].

A further improvement is to take into consideration the effects of temperature on the battery's response by making the model component values a function of temperature. There does not seem to be a consensus on the best way to do this, and each group takes a slightly different approach.

Gao et al. [26] introduce correction factors to the battery capacity and open circuit voltage of the battery that can be calculated from measurements or manufacturers' data sheets. Erdinc et al. [28] use the same voltage correction technique and also models capacity fade with cycle number and battery age considering storage temperature. Verbrugge and Conell [9] have the open circuit voltage, resistors, and self-discharge depend on temperature. The model of Baronti et al. [27] has the resistors depend on temperature, and the model by Lam et al. [31] has the resistors, capacitors, and usable capacity depend on temperature. Hu et al. [30] use 2D splines to make the components depend on both SOC and temperature, and Ceraolo [34] has the circuit components, battery capacity, and open circuit voltages depend on temperature. Some papers [9, 26, 34] consider the battery's thermal generation and heat transfer aspects, while others [27, 28, 30, 31] consider temperature to be an external variable. 
A rarely discussed modelling feature is the fact that the battery's model parameters change depending on whether the battery is being charged or discharged. Aside from the diodes used in the simple circuit models $[11,16,35]$ mentioned at the beginning of the section, very few papers take into consideration the effects of current direction. Hu et al. $[29,30]$ use two completely different sets of battery parameters for the charging and discharging behaviour, requiring the computer simulation to determine which set should be used.

Some models incorporate a capacitor to account for the charge storage capacity of the battery. Since the voltage across a capacitor is the first time integral of the current flowing through it, this can immediately be mapped onto the state of charge. However the open circuit voltage of the battery is not a linear function of the state of charge, so the charge storage capacitor's voltage must be mapped non-linearly onto the open circuit voltage $[23,24,27,28,31,32,44]$.

By putting a resistor in parallel with the charge storage capacitor, the battery's self-discharge can be modelled. However since the time scale of self-discharge is much greater than that of the dynamic effects that are usually of interest, and battery powered vehicles are frequently recharged, the effect of self-discharge can usually be neglected in automotive applications [24, 31].

A circuit diagram of such a model can be seen in Figure C.4, which is based on the model by Chen and Rincón-Mora [24]. The dashed lines indicate the mathematical relationship between the two separate capacity and time-response circuits. Current flowing in the time-response circuit is made to flow in the capacity circuit, while the values of the resistors and capacitors in the time-response circuit are related non-linearly to the voltage of the capacity circuit, which is proportional to the SOC.

To improve this model, Zhang et al. [32] use a discharge-current-dependent charge storage capacitor to model the "C-rate effect", which causes the capacity of the battery to diminish with increasing discharge current. Kim and Qiao [45] take a different approach for modelling the C-rate effect, and replace the charge storage capacitor with a component based on the Kinetic Battery Model (KiBaM) by Manwell and McGowan [53].

The model by Erdinc et al. [28] models the $\mathrm{SOH}$ by taking into consideration the effects of capacity fading and increased series resistance with increasing time and cycle number.

Turning back to general Thévenin circuit models, Hu et al. [36] provide a comparison of different circuit-based Li-ion battery models trained using the same battery data, and discovered that for the LiNMC and $\mathrm{LiFePO}_{4}$ batteries they tested, a battery model containing a single RC parallel pair was sufficient to accurately describe the battery behaviour. In the case of the $\mathrm{LiFePO}_{4}$ battery, adding hysteresis to the model improved the accuracy.

Zhang and Chow [10] performed an investigation into the optimal number of RC parallel pairs that balance the conflicting requirements of high model fidelity and low computational expense, and found that two RC parallel pairs were a good compromise.

Some models are designed to be coupled with an actual battery in order to inform the user about the battery's internal states. For SOC there is the sliding mode observer model by Kim [21], the on-line non-linear regression model of Verbrugge and Tate [20], and the EKF model by Vasebi et al. [17]. For both SOC and SOH there is the non-linear observer model by Bhangu et al. [15]. 


\subsection{Impedance Models}

A special type of circuit-based model is one based on the experimental technique of electrochemical impedance spectroscopy (EIS) [43, 54]. This injects a range of low frequency AC current (up to a few kilohertz) into the battery and measures the battery's voltage response. The magnitude and phase of the response can then be interpreted to give the battery's complex (real and imaginary) impedance as a function of frequency. This method has the limitation that it is a small-signal method that assumes a linear battery model [37] (this fact is actually an asset in the analytical electrochemical models of Sikha and White $[55,56]$ mentioned at the end of Section 4). These models are not often used as a stand-alone simulation of a battery. They are often used to determine the $\mathrm{SOC}$ or $\mathrm{SOH}$ of a resting battery, or are used as a system model in a Kalman filter to provide on-line battery state estimation.

A battery half-cell, representing one half of the battery's chemical reaction that occurs at one of the electrodes, can be modelled by a Randles circuit similar to Figure C.5 [39], where $\mathrm{L}, \mathrm{R}_{\mathrm{i}}$, and $\mathrm{C}$ model the inductance, internal resistance, and capacitance, respectively. The non-linear resistor $\mathrm{R}$ depends on the current flowing through it. The impedance $Z_{w}$, described by Equation 3, refers to a constant-phase Warburg element used to model diffusion, where $A_{w}$ is the Warburg coefficient, $\omega$ is the angular frequency, and $j$ is the imaginary number $\sqrt{-1}$.

$$
Z_{w}(\omega)=\frac{A_{w}}{\sqrt{\omega}}(1-j)
$$

By measuring the complex impedance of the battery as a function of frequency and looking at the spectrum, one can determine the type of electrical circuit network needed to reproduce its behaviour [54]. By using a reference electrode, one can measure and model the spectra of the positive and negative electrodes separately. This gives the most accurate results, since the parameters of both electrodes cannot be reliably determined by measuring them both together [43]. Different battery chemistries can have considerably different EIS spectra. Shih and Lo [54] provide a technical report with many different EIS circuit models that correspond to a wide range of battery chemistries.

Although circuit models abstract away the electrochemical physics happening in the battery, EIS models stay closer to mapping the physics onto circuit elements. Troltzsch et al. [42] provide a nice explanation of how the battery physics maps onto EIS components, and how these effects show up in the EIS spectra. They also describe a clever method of using multiple excitation frequencies and frequency-domain analysis to speed up the spectroscopy procedure.

The EIS spectra, a depiction of which can be seen in Figure C.6, depend on SOC, current, temperature, and $\mathrm{SOH}$. In some ways this is a disadvantage, as it increases the complexity of the model. Huet [43] discusses some of the issues with the temperature dependence of the spectra in relation to determining $\mathrm{SOC}$ and $\mathrm{SOH}$ of batteries in the field.

However it can also be an advantage, as these effects can be included in the model. Models like $[7,19,42]$ are designed to determine SOC and SOH in conjunction with a real battery under test. Others can be used to simulate a stand-alone battery, including various effects on the model behaviour. References [22, 38, 39] consider SOC only, [7] considers $\mathrm{SOC}$ and $\mathrm{SOH}$, while [8] considers $\mathrm{SOC}, \mathrm{SOH}$, and temperature. 
Using a complex non-linear least squares fitting algorithm [57] seems to be the usual way of determining the circuit model parameters from EIS spectra. Troltzsch et al. [42] use a combination of a genetic global optimization and Levenberg-Marquardt local optimization to find model parameters.

Another challenge is that the behaviour of the Warburg element is defined in the frequency domain, and must be converted to a series of resistor-capacitor pairs in order to be simulated in the time domain [22, 38]. Increasing the number of pairs increases the model fidelity, but also the amount of computation time required.

\section{Electrochemical Models}

Unlike equivalent circuit modelling approaches, electrochemical models explicitly represent the chemical processes that take place in the battery. These models describe the battery processes in great detail, making them the most accurate of battery models. However, the highly detailed description results in considerable computational complexity, and it may take hours to simulate a charge-discharge cycle of a detailed battery model if no model reduction approach is used to treat the battery equations.

There are several approaches to developing electrochemical battery models. One of the most popular approaches is a lumped parameter model where the battery is described by a small set of differential algebraic equations (DAEs) that represent the time-dependent electrochemical phenomena between the electrodes, while assuming a uniform spatial distribution of chemical products. This approach constitutes the majority of the early physics-based battery models, mostly for describing simple lead-acid and non-rechargeable batteries [58, 59]. This approach is based on Nernst's theory and the Butler-Volmer equation. For example, the equation for the open-circuit potential that relates the electrical potential $\Phi(t)$ of an electrode to the electrical charge or molality $m(t)$ for a galvanic cell can be described using Nernst's equation [58] as:

$$
\Phi(t)=\Phi^{0}-\frac{R T}{n F} \ln \left(\prod_{k}\left(m_{k}(t) \gamma_{k}\right)^{\nu_{k}}\right)
$$

in which the $k$ subscript represents the $k^{\text {th }}$ reactant, $m$ is the molality, $\gamma$ is the activity coefficient, $n$ is the number of electrons, $\nu$ is the stoichiometric coefficient, and $\Phi^{0}$ is the electromotive force at standard conditions. Please see Appendix B for the nomenclature used in these chemistry equations.

The Butler-Volmer equation describes the relationship between battery current density $J(t)$ and over-potential $\eta(t)$ (alternatively called polarization) by

$$
J(t)=J_{0}\left[\exp \left(\frac{\alpha F}{R T} \eta(t)\right)-\exp \left(\frac{(1-\alpha) F}{R T} \eta(t)\right)\right]
$$

where $\alpha$ is called the charge transfer coefficient and $J_{0}$ is the exchange current density.

These two equations constitute most of the early primary and secondary battery models, mostly for lead-acid batteries [58] and some NiMH cells [60, 61]. An advantage of the lumped models is that the equations are fairly simple and easy to integrate, resulting in a fast and accurate model suitable for automotive applications and control design purposes. As an example, the full NiMH battery model developed by Wu et al. 
[60] simulates in milliseconds using today's computers. When reorganized using a linear graph theory formulation that results in a more optimized and compact set of equations, the simulation time reduces a further $30 \%$ [61].

The main drawback of the lumped parameter approach is that the simple DAEs are insufficient to describe the complex electrochemical processes in most modern batteries produced using today's advanced technology, such as Li-ion cells. Rapid advances in battery research, resulting in new electrode materials and battery designs, requires better models that can describe more complex electrochemical and physical processes in greater detail. For example, one needs to model the spatial distribution of $\mathrm{Li}+$ ions and the electrical potential between the two electrodes to have an insight into the temperature distribution in a Li-ion cell or an HEV battery pack. This is where the lumped models fall short and a distributed model is needed to meet such requirements. Newman et al. [62-65] pioneered the development of porous electrode theory to describe models for Li-ion cells. This method has become the foundation and standard for most physicsbased battery modelling techniques today and, in many cases, is used for generating battery testing data for model validations. This model describes a battery in great detail and considers all the major electrochemical processes including mass transport and diffusion, ion distribution, side reactions, temperature effects, and battery ageing. Most of the current rigorous battery models are derived from the porous electrode and concentrated solution theories that describe charge, discharge, and species transport in the solid and electrolyte phases across a simplified one-dimensional spatial cell structure. This 1D model considers dynamics along only one axis (the horizontal $x$-axis shown in Figure C.7) and neglects the dynamics along the remaining two axes ( $y$-axis and $z$-axis) $[62,63,66-70]$. This approximation is applicable to most cell structures as the length scale of a typical Li-ion cell along the $x$-axis is on the order of $100 \mu m$, whereas the length scale for the remaining two axes is on the order of 100,000 $\mu \mathrm{m}$ or more [71].

In contrast to lumped-parameter modelling, the porous electrode theory uses partial differential equations (PDEs) to describe the electrochemical processes. In general, these PDEs are derived based on Fick's law of diffusion for the active material concentration, Ohm's law for electrical potential distributions, and the Nernst and Butler-Volmer equations. Specifically, the PDEs that describes the changes in lithium concentration in solid and liquid phases due to the gradient changes in the diffusive flow of $\mathrm{Li}+$ ions are given by:

$$
\frac{\partial c_{\mathrm{s}, k}(x, r, t)}{\partial t}=\frac{D_{\mathrm{s}, k}}{r^{2}} \frac{\partial}{\partial r}\left(r^{2} \frac{\partial c_{\mathrm{s}, k}(x, r, t)}{\partial r}\right)
$$

and

$$
\epsilon_{k} \frac{\partial c_{\mathrm{e}, k}(x, t)}{\partial t}=\frac{\partial}{\partial x}\left(D_{\mathrm{eff}, k} \frac{\partial c_{\mathrm{e}, k}(x, t)}{\partial x}\right)+a_{k}\left(1-t_{+}\right) J_{k}(x, t)
$$

where $k=\mathrm{p}$ for the positive electrode and $k=\mathrm{n}$ for the negative electrode.

Charge conservation in the solid phase of each electrode can be described by Ohm's law

$$
\sigma_{\mathrm{eff}, k} \frac{\partial^{2} \Phi_{\mathrm{s}, k}(x, t)}{\partial x^{2}}=a_{k} F J_{k}(x, t)
$$

with boundary conditions at the current collectors being proportional to the applied 
current density

$$
\begin{gathered}
-\left.\sigma_{\mathrm{eff}, \mathrm{p}} \frac{\partial \Phi_{\mathrm{s}, \mathrm{p}}}{\partial x}\right|_{x=0}=-\left.\sigma_{\mathrm{eff}, \mathrm{n}} \frac{\partial \Phi_{\mathrm{s}, \mathrm{n}}}{\partial x}\right|_{x=L}=I \\
-\left.\sigma_{\mathrm{eff}, \mathrm{p}} \frac{\partial \Phi_{\mathrm{s}, \mathrm{p}}}{\partial x}\right|_{x=L_{\mathrm{p}}}=-\left.\sigma_{\mathrm{eff}, \mathrm{n}} \frac{\partial \Phi_{\mathrm{s}, \mathrm{n}}}{\partial x}\right|_{x=L_{\mathrm{p}}+L_{\mathrm{s}}}=0
\end{gathered}
$$

where the current density $I$ is related to the applied current $i$ and the surface area $A$ of the electrode as $I=i / A$.

The effective electronic conductivity can be expressed in terms of the porosity of the electrode as

$$
\sigma_{\mathrm{eff}, k}=\sigma_{k}\left(1-\epsilon_{k}-\epsilon_{\mathrm{f}, k}\right)
$$

Combining Kirchhoff's law with Ohm's law in the electrolyte phase yields

$$
-\sigma_{\mathrm{eff}, k} \frac{\partial \Phi_{\mathrm{s}, k}(x, t)}{\partial x}-\kappa_{\mathrm{eff}, k} \frac{\partial \Phi_{\mathrm{e}, k}(x, t)}{\partial x}+\frac{2 \kappa_{\mathrm{eff}, k}(x, t) R T}{F}\left(1-t_{+}\right) \frac{\partial \ln c_{\mathrm{e}, k}}{\partial x}=I
$$

The Butler-Volmer equation describing the relationship between the current density, concentrations, and over-potential is given by [68]

$$
\begin{aligned}
J_{k}(x, t)= & K_{k}\left(c_{\mathrm{s}, k, \max }-c_{\mathrm{s}, k, \text { surf }}\right)^{0.5}\left(c_{\mathrm{s}, k, \text { surf }}\right)^{0.5} c_{\mathrm{e}, k}^{0.5}(x, t) \\
& \times\left[\exp \left(\frac{0.5 F}{R T} \mu_{\mathrm{s}, k}(x, t)\right)-\exp \left(-\frac{0.5 F}{R T} \mu_{\mathrm{s}, k}(x, t)\right)\right]
\end{aligned}
$$

These partial differential algebraic equations (PDAEs) are defined separately for each of the positive electrode, separator, and negative electrode regions and are coupled with each other by the continuity in the boundary conditions. In total, there are 14 non-linear PDAEs with 14 unknowns.

The traditional methods for solving battery PDEs were mainly based on finite difference technique, which approximates continuous quantities as being constant within discrete evenly-spaced intervals along the $x$ axis, and approximates the derivatives based on a Taylor series expansion. As an example, the second derivative of the $\mathrm{Li}^{+}$concentration in liquid phase can be approximated as

$$
\frac{\partial^{2} c_{\mathrm{e}, k}(x, t)}{\partial x^{2}}=\frac{c_{i+1}(t)-2 c_{i}(t)+c_{i-1}(t)}{h^{2}}
$$

where segments along the $x$ axis are indexed with $i$, and $h$ is the length of a segment. When this is substituted into Equation (7) it results in

$$
\epsilon_{k} \frac{\partial c_{\mathrm{e}, k}(x, t)}{\partial t}=D_{\mathrm{eff}, k} \frac{c_{i+1}(t)-2 c_{i}(t)+c_{i-1}(t)}{h^{2}}+a_{k}\left(1-t_{+}\right) J_{i}(t)
$$

The boundary conditions can be approximated using forward and backward difference forms as

$$
\begin{gathered}
\left.\frac{\partial c_{\mathrm{e}, k}(x, t)}{\partial x}\right|_{x=0}=\frac{c_{i+1}(t)-c_{i}(t)}{h} \\
\left.\frac{\partial c_{\mathrm{e}, k}(x, t)}{\partial x}\right|_{\substack{x=L_{\mathrm{p}} \\
11}}=\frac{c_{i}(t)}{c_{i-1}(t)}
\end{gathered}
$$


The end result is a set of $N$ linear differential equations, with $N$ being the number of discretization segments. The finite difference method is simple, yet to obtain good accuracy $N$ must be quite large due to the loss of precision when non-linear equations are linearly approximated. This makes solving the whole battery system in real-time difficult using the finite difference method. During the last few years, many model reduction methods have been developed around this porous-electrode theory to make the battery models suitable for real-time applications. These approaches can be classified into two different groups: (1) trying to reduce the order of the model while preserving the fidelity of the system, and (2) trying to neglect some the model properties with a loss of information.

The first group consists of mathematical techniques to better handle the model PDEs or to convert the PDEs into a simpler form that can be integrated faster using numerical solvers. Some of the most well-known methods are based on temporal-spatial separation techniques, including collocation method and Galerkin's method. The idea behind these methods is similar and is based on finding the approximate numerical solution to a nonlinear PDE using a set of orthogonal basis functions which turns the PDE into coupled set of ODEs. The real utility of these methods comes from their application to nonlinear PDEs. Take the liquid phase concentration PDE in Equation (7) as an example, the Galerkin idea is to start off with the spatial decomposition and allow time-varying coefficients:

$$
\phi(x, t)=\sum_{j=1}^{N} \eta_{j}(t) \alpha_{j}(x)
$$

where $N$ is the number of node points, $\alpha_{j}(x)$ are the orthogonal basis functions that have been carefully chosen to satisfy the boundary condition of (7), and $\eta_{j}$ are the unknown functions of time to be solved for. Chebychev polynomials or sine functions are good candidates for the basis function. Let's assume that $\alpha_{j}(x)=\cos \left(\frac{j \pi}{L} x\right)$. Inserting this approximate solution into the full PDE in (7) gives

$$
R_{c}(x, t)=\epsilon_{k} \sum_{j=1}^{N} \alpha_{j}(x) \frac{\mathrm{d} \eta_{j}(t)}{\mathrm{d} t}-D_{\text {eff }, k} \sum_{j=1}^{N} \frac{\mathrm{d}^{2} \alpha_{j}(x)}{\mathrm{d} t^{2}} \eta_{j}(t)+a_{k}\left(1-t_{+}\right) J \approx 0
$$

This function is known as the residual. In Galerkin's method, we replace the condition that the residual should be approximately zero by the condition that the residual should be orthogonal to the set of basis functions. That is, for $j=0, \ldots, N$ we multiply the residual by the basis function $\cos \left(\frac{j \pi}{L} x\right)$ and integrate it over $x$, then set the result to zero. In the collocation method, we choose a finite set of points where the exact solutions must be matched. These points are called collocation points and at these points, the residual becomes zero. The collocation and Galerkin's techniques both result in a set of ODEs which, when solved, give the approximate numerical solution of the time-varying unknowns. These unknowns can be substituted into the approximate solution in (18) to obtain the order-reduced form of the PDE.

The techniques outlined above have been used in the works of Subramanian et al. in [72-75]. As one of the pioneers in battery modelling, Subramanian et al. developed a 
wide range of methods for battery model reduction and a real-time simulation model using a combination of perturbation techniques and heuristic simplifications [72-74]. They reported that the wall time for their real-time simulation model for a single process was around $100 \mathrm{~ms}$ to simulate a complete charge-discharge cycle. To derive the lower-order model using this method, one needs to carry out preprocessing and have a priori knowledge of the behaviour of the system under different conditions, which makes their method less flexible than desired. In recent research, Ramadesigan et al. expanded Subramanian's collocation method to simulate the electrochemical-thermal behaviours of a battery pack in real-time [75] and used Subramanian's reformulated model for estimation of battery capacity fade (decrease in $\mathrm{SOH}$ ) [76].

Other methods, including the Chebyshev polynomial method [77, 78], the residue grouping method [79, 80], proper orthogonal decomposition method [81], and Padé approximation [82] have also been used to derive reduced-order models for Li-ion batteries. In the methods using Chebyshev polynomials, the state variables are approximated by linear combinations of several Chebyshev polynomials, and then an approximate model is projected onto a subspace formed by these orthogonal Chebyshev polynomials to form a reduced-order model, which can then be solved for the unknown coefficients in the truncated expressions. With the residue grouping method, Smith et al. were able to reduce computational time by roughly 20 times compared to the full-order model [79]. Smith et al. [83] also developed a control-oriented one-dimensional (1D) electrochemical model by using the method of residue grouping. Their transfer functions are represented by a truncated series of grouped residues with similar eigenvalues. Cai and White [81] proposed an approach based on proper orthogonal decomposition for tackling the problem by using two steps of approximation: PDE discretization, and truncation of the number of orthogonal modes. Cai and White showed that the order-reduced model simulated seven times faster than the full-order model for a similar level of accuracy. Some of the most recent efforts for model reduction in this category include the work of Dao et al. [84], who applied Galerkin's method with sinusoidal shape functions that has resulted in a fast model suitable for real-time vehicle applications. Dao et al. also applied the simplified battery models to a number of high-fidelity EV and HEV systems [85] which simulated several orders of magnitude faster than real-time.

Most recently, Guo and White [86] and Hu et al. [87] applied model reduction techniques to reformulate Li-ion equations as a state-space model for system identification. Their models can be applied for both spherical and non-spherical particles using a transfer function calculation based on step response and complex exponential methods. Guo and White [86] showed that the approximate solution can provide accuracy and timeefficiency in simulation. They also indicated that the approximate solution simulates faster at high current rates and shows better long-time accuracy than the short-time solution.

The methods outlined above result in fast models with little loss of information and are, therefore, very suitable for applications that require high model fidelity. However, not all applications require high fidelity over a wide range of battery operating conditions. For example, many applications only need model accuracy with low-to-medium charge/discharge currents. In this case, techniques such as the electrode averaging method [88] or volume-averaging technique [89] can be used to produce a fast chemistrybased model. Speltino et al. also made several simplifications such as neglecting solid concentration distribution and assuming constant electrolyte concentration. As a result, 
their model simulated quickly, but with a heavy loss of information [88, 90].

An interesting application of porous electrode theory to EIS is in the papers of Sikha and White $[55,56]$ where they develop analytical expressions for the impedance spectrum of a Li-ion cell.

Due to the small signal amplitude used in EIS, they are able to use a small-signal approximation and solve the electrochemical equations in the Laplace domain. With this they get a closed-form analytical expression for the impedance spectrum that sheds light on the contribution of the different physical processes on the resulting spectrum, and allows the spectrum to be calculated under different limiting conditions.

Their model produces results that are very close to numerical simulations, and are an order of magnitude faster. Due to the accuracy of the model, physical parameters of a battery can be estimated using experimental data.

\section{Simulation Example}

The parameters for both circuit and electrochemical models can be tuned so that the simulations produce very similar results. An equivalent circuit model is usually easier to build, but might be more difficult to expand and incorporate some of the battery physical behaviours. Hence, one has to decide what battery model to use depending on the type of application. As a comparison between the two modelling approaches, the simulation results from both equivalent circuit and electrochemical models are shown in Figure C.8, and compared to experimental data taken from Figure 5 of [24]. The equivalent circuit model, its parameters, and its current discharge curve are the same as those used by Chen and Rincón-Mora in [24], and the electrochemical model's parameters have been tuned to match it. The sizes of the electrodes on the electrochemical model were tuned to ensure that the two models have the same capacity. See Table D.1 for the parameters used in the electrochemical model, and Appendix A for the parameters used in the equivalent circuit model. We were able to make the two curves match up closely with each other with little effort in parameter tuning.

To investigate the sensitivity of the models to parameter uncertainty or variation, a sensitivity analysis was performed on the equivalent circuit and electrochemical models used to generate Figure C.8. Parameters were assigned to the equivalent circuit model equations presented in Appendix A, yielding the following equations:

$$
\begin{aligned}
C_{\text {cap }} & =P_{1} \\
V_{\text {oc }} & =P_{2} \exp \left(P_{3} V_{\text {soc }}\right)+P_{4}+P_{5} V_{\text {soc }}+P_{6} V_{\text {soc }}^{2}+P_{7} V_{\text {soc }}^{3} \\
R_{\text {series }} & =P_{8} \exp \left(P_{9} V_{\text {soc }}\right)+P_{10} \\
R_{\text {short }} & =P_{11} \exp \left(P_{12} V_{\text {soc }}\right)+P_{13} \\
C_{\text {short }} & =P_{14} \exp \left(P_{15} V_{\text {soc }}\right)+P_{16} \\
R_{\text {long }} & =P_{17} \exp \left(P_{18} V_{\text {soc }}\right)+P_{19} \\
C_{\text {long }} & =P_{20} \exp \left(P_{21} V_{\text {soc }}\right)+P_{22}
\end{aligned}
$$

Parameters were also assigned to the electrochemical model. The non-constant parameters that had non-zero sensitivities were assigned the parameter names appearing in Table D.2. 
The model sensitivities were found using the RMS difference between the simulated voltage, $V_{\operatorname{sim}}(t)$, and the experimental voltage, $V_{\exp }(t)$, using the following equation:

$$
R M S=\sqrt{\int_{0}^{t_{\text {end }}}\left(V_{\text {sim }}(t)-V_{\exp }(t)\right)^{2} d t}
$$

and the sensitivity for parameter $\nu$ is defined as:

$$
\mu_{\nu}=\frac{R M S_{p}-R M S_{0}}{R M S_{0}} \frac{\nu_{0}}{\nu_{p}-\nu_{0}}
$$

where $\nu_{p}$ and $\nu_{0}$ are the perturbed and nominal parameter values, respectively, and $R M S_{p}$ and $R M S_{0}$ are RMS values corresponding to the perturbed and nominal $\nu$ values, respectively. The sensitivity represents the fraction change in the RMS fit of the simulation to the experimental data given a fraction change in one of the parameters of the simulation.

By using $\nu_{p}=1.01 \nu_{0}$ for each of the parameters in the equivalent circuit and electrochemical models, and $t_{\text {end }}=20000 \mathrm{~s}$ for the simulation, Figure C.9 was generated, and used the data from Figure 5 of [24] as the experimental data. Parameters that had zero sensitivity were omitted from the figure.

As can be seen from Figure C.9, the parameters of greatest sensitivity for the equivalent circuit model are the total battery capacitance and the parameters expressing the polynomial component of the open circuit voltage, followed by the constant parts in the expressions of the resistor and capacitor components. The parameters in the exponential functions are of much lower sensitivity than the others.

For the electrochemical model the electrode area, thickness, radius of intercalation, volume fraction, and maximum $\mathrm{Li}^{+}$ion concentration for the positive and negative electrodes were the most important, with the separator being significantly less sensitive.

In $\mathrm{EV} / \mathrm{HEV}$ simulations, the Li-ion models simulate quickly, as anticipated. As an example, we simulated an EPA drive cycle with a series HEV model comprising a meanvalue engine, power control unit, complete cooling system, and battery pack, a block diagram of which can be seen in Figure C.10. The battery pack consisted of 70 Li-ion cells which were modelled from the rigorous PDAE, and the results of the simulation are plotted in Figure C.11.

Using a 2.0 GHz PC laptop computer and a Runge-Kutta numeric integrator with 1 $\mathrm{ms}$ time step took 12 seconds to simulate in MapleSim ${ }^{\mathrm{TM}} 6.0$.

The execution time is reduced largely due to the symbolic model reduction techniques performed by MapleSim, which reduces the number of symbolic equations from 874 to 79 with no loss of fidelity.

\section{Conclusions}

Battery modelling is a broad and complicated field, with no single model capable of meeting the requirements for all applications.

Two of the most common techniques, equivalent-circuit modelling and electrochemical modelling, were discussed in detail, and battery models suitable for real-time simulation, control systems, battery state estimation, state of health, thermal effects, and highfidelity modelling were touched upon. 
Which model to use in one's application depends on the application constraints and the specific quantities of interest one is interested in simulating.

\section{Acknowledgements}

We would like to acknowledge the support of the Natural Sciences and Engineering Research Council (NSERC) of Canada, Toyota, and MapleSoft.

\section{References}

[1] R. Rao, S. Vrudhula, D. N. Rakhmatov, Battery modeling for energy-aware system design, Computer 36 (12) (2003) 77-87.

[2] M. Pedram, Q. Wu, Design considerations for battery-powered electronics, Proc. Design Automation Conference (1999) 861-866.

[3] M. Jongerden, B. Haverkort, Which battery model to use?, IET Software 3 (6) (2009) $445-457$.

[4] D. W. Gao, C. Mi, A. Emadi, Modeling and simulation of electric and hybrid vehicles, in: Proc. IEEE, Vol. 95, 2007, pp. 729-745.

[5] S. M. Lukic, J. Cao, R. C. Bansal, F. Rodriquez, A. Emadi, Energy storage systems for automotive applications, IEEE Trans. Ind. Elec. 55 (6) (2008) 2258-2267.

[6] A. Pesaran, Battery choices and potential requirements for plug-in hybrids, Tech. rep., National Renewable Energy Laboratory (February 2007).

[7] E. Prada, J. Bernard, V. Sauvant-Moynot, Ni-MH battery ageing: from comprehensive study to electrochemical modelling for state-of charge and state-of-health estimation, in: Engine and Powertrain Control, Simulation and Modeling, 2009.

[8] E. Prada, D. D. Domenico, Y. Creff, J. Bernard, V. Sauvant-Moynot, A coupled 0D electrochemical ageing electro-thermal li-ion modeling approach for HEV/PHEV, in: Vehicle Power and Propulsion Conference, 2011, pp. 1-8.

[9] M. W. Verbrugge, R. S. Conell, Electrochemical and thermal characterization of battery modules commensurate with electric vehicle integration, Journal of the Electrochemical Society 149 (1) (2002) A45-A53.

[10] H. Zhang, M.-Y. Chow, Comprehensive dynamic battery modeling for phev applications, in: Power and Energy Society General Meeting, 2010 IEEE, 2010, pp. 1 -6. doi:10.1109/PES.2010.5590108.

[11] B. K. Powell, T. E. Pilutti, A range extender hybrid electric vehicle dynamic model, in: Proceedings of the 33rd Conference on Decision and Control, 1994, pp. 2736-2741.

[12] B. K. Powell, K. E. Bailey, S. R. Cikanek, Dynamic modeling and control of hybrid electric vehicle powertrain systems, IEEE Control Systems 18 (5) (1998) 17-33.

[13] S. Abu-Sharkh, D. Doerrfel, Rapid test and non-linear model characterisation of solid-state lithiumion batteries, Journal of Power Sources 130 (2004) 266-274.

[14] M. J. Kim, H. Peng, C.-C. Lin, E. Stamos, D. Tran, Testing, modeling, and control of a fuel cell hybrid vehicle, in: 2005 American Control Conference, 2005, pp. 3859-3864.

[15] B. S. Bhangu, P. Bently, D. A. Stone, C. M. Bingham, Nonlinear observers for predicting state-ofcharge and state-of-health of lead-acid batteries for hybrid-electric vehicles, IEEE Transactions on Vehicular Technology 54 (3) (2005) 783-794.

[16] G. L. Plett, Extended kalman filtering for battery management systems of LiPB-based HEV battery packs part 2. modeling and identification, Journal of Power Sources 134 (2004) 262-276.

[17] A. Vasebi, M. Partovibakhsh, S. M. T. Bathaee, A novel combined battery model for state-ofcharge estimation in lead-acid batteries based on extended kalman filter for hybrid electric vehicle applications, Journal of Power Sources 174 (2007) 30-40.

[18] W. X. Shen, C. C. Chan, E. W. C. Lo, K. T. Chau, Adaptive neuro-fuzzy modeling of battery residual capacity for electric vehicles, IEEE Transactions on Industrial Electronics 49 (3) (2002) $677-684$.

[19] A. J. Salkind, C. Fennie, P. Singh, T. Atwater, D. E. Reisner, Determination of state-of-charge and state-of-health of batteries by fuzzy logic methodology, Journal of Power Sources 80 (1999) 293-300.

[20] M. Verbrugge, E. Tate, Adaptive state of charge algorithm for nickel metal hydride batteries including hysteresis phenomena, Journal of Power Sources 126 (2004) 236-249. 
[21] I.-S. Kim, Nonlinear state of charge estimator for hybrid electric vehicle battery, IEEE Transactions on Power Electronics 23 (4) (2008) 2027-2034.

[22] E. Kuhn, C. Forgez, P. Lagonotte, G. Friedrich, Modelling ni-mh battery using cauer and foster structures, Journal of Power Sources 158 (2006) 1490-1497.

[23] R. C. Kroeze, P. T. Krein, Electrical battery model for use in dynamic electric vehicle simulations, in: Power Electronics Specialists Conference, 2008, pp. 1336-1342.

[24] M. Chen, G. A. Rinćon-Mora, Accurate electrical battery model capable of predicting runtime and I-V performance, IEEE Transactions on Energy Conversion 21 (2) (2006) 504-511.

[25] V. H. Johnson, A. A. Pesaran, T. Sack, Temperature-dependent battery models for high-power lithium-ion batteries, in: 17th Annuel Electric Vehicle Symposium, 2000

[26] L. Gao, S. Liu, R. A. Dougal, Dynamic lithium-ion battery model for system simulation, Components and Packaging Technologies, IEEE Transactions on 25 (3) (2002) 495-505.

[27] F. Baronti, G. Fantechi, E. Leonardi, R. Roncella, R. Saletti, Enhanced model for lithium-polymer cells including temperature effects, in: IECON 2010 - 36th Annual Conference on IEEE Industrial Electronics Society, 2010, pp. 2329 -2333. doi:10.1109/IECON.2010.5675134.

[28] O. Erdinc, B. Vural, M. Uzunoglu, A dynamic lithium-ion battery model considering the effects of temperature and capacity fading, in: Clean Electrical Power, 2009 International Conference on, 2009, pp. 383 -386. doi:10.1109/ICCEP.2009.5212025.

[29] Y. Hu, S. Yurkovich, Y. Guezennec, B. Yurkovich, A technique for dynamic battery model identification in automotive applications using linear parameter varying structures, Control Engineering Practice 17 (10) (2009) 1190 - 1201. doi:10.1016/j.conengprac.2009.05.002.

[30] Y. Hu, S. Yurkovich, Y. Guezennec, B. Yurkovich, Electro-thermal battery model identification for automotive applications, Journal of Power Sources 196 (1) (2011) 449 - 457 doi:10.1016/j.jpowsour.2010.06.037.

[31] L. Lam, P. Bauer, E. Kelder, A practical circuit-based model for li-ion battery cells in electric vehicle applications, in: Telecommunications Energy Conference (INTELEC), 2011 IEEE 33rd International, 2011, pp. 1 -9. doi:10.1109/INTLEC.2011.6099803.

[32] J. Zhang, S. Ci, H. Sharif, M. Alahmad, An enhanced circuit-based model for single-cell battery, in: Applied Power Electronics Conference and Exposition (APEC), 2010 Twenty-Fifth Annual IEEE, 2010, pp. 672 -675. doi:10.1109/APEC.2010.5433597.

[33] B. Schweighofer, K. M. Raab, G. Brasseur, Modeling of high power automotive batteries by the use of an automated test system, IEEE Transactions on Intrumentation and Measurement 52 (4) (2003) 1087-1091.

[34] M. Ceraolo, New dynamical models of lead-acid batteries, IEEE Transactions on Power Systems 15 (4) (2000) 1184-1190.

[35] H. Chan, D. Sutanto, A new battery model for use with battery energy storage systems and electric vehicles power systems, in: Power Engineering Society Winter Meeting, Vol. 1, 2000, pp. 470-475.

[36] X. Hu, S. Li, H. Peng, A comparative study of equivalent circuit models for li-ion batteries, Journal of Power Sources 198 (2012) 359-367.

[37] E. Karden, S. Buller, R. W. D. Doncker, A method for measurements and intrepretation of impedance spectra for industrial batteries, Journal of Power Sources 85 (2000) 72-78.

[38] S. Buller, M. Thele, R. W. D. Doncker, E. Karden, Impedance-based simulation models of supercapacitors and li-ion batteries for power electronic applications, IEEE Transactions on Industry Applications 41 (3) (2005) 742-747.

[39] S. Buller, M. Thele, E. Karden, R. W. D. Doncker, Impedance-based non-linear dynamic battery modeling for automotive applications, Journal of Power Sources 113 (2003) 422-430.

[40] J. Wang, K. Zou, C. Chen, L. Chen, A high frequency battery model for current ripple analysis, in: Applied Power Electronics Conference and Exposition (APEC), 2010 Twenty-Fifth Annual IEEE, 2010, pp. 676 -680. doi:10.1109/APEC.2010.5433598.

[41] D. Linden, T. B. Reddy, Handbook of Batteries, 3rd Edition, McGraw-Hill, 2001.

[42] U. Tröltzsch, O. Kanoun, H.-R. Tränkler, Characterizing aging effects of lithium ion batteries by impedance spectroscopy, Electrochimica Acta 51 (89) (2006) $1664 \quad$ - 1672. doi:10.1016/j.electacta.2005.02.148.

[43] F. Huet, A review of impedance measurements for determination of the state-of-charge or state-ofhealth of secondary batteries, Journal of Power Sources 70 (1998) 59-69.

[44] J. Zhang, J. Lee, A review on prognostics and health monitoring of li-ion battery, Journal of Power Sources 196 (15) (2011) 6007 - 6014. doi:10.1016/j.jpowsour.2011.03.101.

[45] T. Kim, W. Qiao, A hybrid battery model capable of capturing dynamic circuit characteristics and nonlinear capacity effects, Energy Conversion, IEEE Transactions on 26 (4) (2011) $1172-1180$. 
doi:10.1109/TEC.2011.2167014.

[46] T. Hu, B. Zanchi, J. Zhao, Simple analytical method for determining parameters of discharging batteries, Energy Conversion, IEEE Transactions on 26 (3) (2011) 787 -798. doi:10.1109/TEC.2011.2129594.

[47] S. Gold, A PSPICE macromodel for lithium-ion batteries, in: Twelfth Annual Battery Conference on Applications and Advances, 1997, pp. 215-222.

[48] S. C. Hageman, Simple PSpice models let you simulate commmon battery types, EDN 38 (1993) $117-132$.

[49] S. Piller, M. Perrin, A. Jossen, Methods for state-of-charge determination and their applications, Journal of Power Sources 96 (2001) 113-120.

[50] V. Pop, H. J. Bergveld, P. H. L. Notten, P. P. L. Regtien, State-of-the-art of battery state-of-charge determination, Measurement Science and Technology 16 (12) (2005) R93.

[51] A. Szumanowski, Y. Chang, Battery management system based on battery nonelinear dynamics modeling, IEEE Transactions on Vehicular Technology 57 (3) (2008) 1425-1432.

[52] T. F. Fuller, M. Doyle, J. Newman, Relaxation phenomena in lithium-ion-insertion cells, J. Electrochem. Soc. 141 (4) (1994) 982-990.

[53] J. F. Manwell, J. G. McGowan, Lead acid battery storage model for hybrid energy systems, Solar Energy 50 (5) (1993) 399 - 405. doi:10.1016/0038-092X(93)90060-2.

[54] H. Shih, T.-C. Lo, Electrochemical impedance spectroscopy for battery research and development, Tech. rep., Solartron Instruments (1996).

[55] G. Sikha, R. E. White, Analytical expression for the impedance response of an insertion electrode cell, Journal of The Electrochemical Society 154 (1) (2007) A43-A54.

[56] G. Sikha, R. E. White, Analytical expression for the impedance response for a lithium-ion cell, Journal of The Electrochemical Society 155 (12) (2008) A893-A902.

[57] J. R. Macdonald, LEVM complex nonlinear least squares fitting program.

[58] H. Bode, Lead Acid Batteries, Wiley and Sons, 1977.

[59] I. Levine, Physical Chemistry, McGraw-Hill, 1988.

[60] B. Wu, M. Mohammed, D. Brigham, R. Elder, R. White, A non-isothermal model of a nickel-metal hydride cell, Journal of Power Sources 101 (2) (2001) 149-157.

[61] T.-S. Dao, J. McPhee, Dynamic modeling of electrochemical systems using linear graph theory, Journal of Power Sources 196 (23) (2011) 10442-10454.

[62] J. Newman, T. William, Porous-electrode theory with battery applications, AIChE Journal 21 (1) (1975) 25-41.

[63] M. Doyle, T. Fuller, J. Newman, Modeling of galvanostatic charge and discharge of the lithium/polymer/insertion cell, J. Electrochem Soc 140 (1993) 1526-33.

[64] M. Doyle, J. Newman, A. Gozdz, C. Schmutz, J. Tarascon, Comparison of modeling predictions with experimental data from plastic lithium ion cells, J. Electrochem. Soc. 143 (6) (1996) 1890-1903.

[65] C. M. Doyle, Design and simulation of lithium rechargable batteries, Ph.D. thesis, University of California at Berkeley (1995).

[66] T. Fuller, M. Doyle, J. Newman, Simulation and optimization of the dual lithium ion insertion cell, J. Electrochem Soc 141 (1994) 1-10.

[67] K. Thomas, J. Newman, R. Darling, Advances in Lithium-Ion Batteries: Mathematical Modeling of Lithium Batteries, Springer-Verlag, New York, 2002.

[68] J. Newman, K. Thomas-Aleya, Electrochemical Systems, 3rd Edition, Wiley and Sons, 2004

[69] I. Ong, J. Newman, Double-layer capacitance in a dual lithium ion insertion cell, J. Electrochem. Soc. 146 (12) (1999) 4360-4365.

[70] P. Gomadam, J. Weidner, R. Dougal, R. White, Mathematical modeling of lithium-ion battery systems, Journal of Power Sources 110 (2002) 267-284.

[71] N. Chaturvedi, R. Klein, J. Christensen, J. Ahmed, A. Kojic, Modeling, estimation, and control challenges for lithium-ion batteries, IEEE Control Systems Magazine (2010) 1997-2002.

[72] V. Subramanian, V. Boovaragavan, V. Diwakar, Toward real-time simulation of physics based lithium-ion battery models, Electrochemical and Solid-State Letters 10 (11) (2007) A255-A260.

[73] V. Subramanian, V. Boovaragavan, V. Ramadesigan, M. Arabandi, Mathematical model reformulation for lithium-ion battery simulations: galvanostatic boundary conditions, Journal of the Electrochemical Society 156 (4) (2009) A260-A271.

[74] V. Ramadesigan, V. Boovaragavan, J. Pirkle, V. Subramanian, Efficient reformulation of solidphase diffusion in physics-based lithium-ion battery models, Journal of the Electrochemical Society 157 (7) (2010) A854-A860.

[75] P. Northrop, V. Ramadesigan, S. De, V. Subramanian, Coordinate transformation, orthogonal 
collocation and model reformulation for simulating electrochemical-thermal behavior of lithium-ion battery stacks, J. Electrochem. Soc. 158 (12) (2011) A1461-A1477.

[76] V. Ramadesigan, K. Chen, N. Burns, V. Boovaragavan, R. Braatz, V. Subramanian, Parameter estimation and capacity fade analysis of lithium-ion batteries using reformulated models, J. Electrochem. Soc. 158 (9) (2011) A1048-A1054.

[77] M. Golubitky, D. Schaeffer, Singularities and Groups in Bifurcation Theory, Vol. 1, Springer-Verlag, Berlin, 1984.

[78] B. Bhikkaji, T. Soderstrom, Reduced order models for diffusion systems using singular pertubations, Energy and Buildings 33 (8) (2001) 769-781.

[79] K. Smith, C. Rahn, C. Wang, Model order reduction of 1D diffusion systems via residue grouping, Journal of Dynamic Systems, Measurement, and Control 130.

[80] K. Smith, C. Rahn, C. Wang, Model-based electrochemical estimation and constraint management for pulse operation of lithium ion batteries, IEEE Trans. on Control Systems Technology 18 (3) (2010) 654-663.

[81] L. Cai, R. White, Reduction of model order based on proper orthogonal decomposition for lithiumion battery simulations, Journal of The Electrochemical Society 156 (3) (2009) A154-A161.

[82] J. Forman, S. Bashash, J. Stein, H. Fathy, Reduction of an electrochemistry-based li-ion battery model via quasi-linearization and padé approximation, Journal of The Electrochemical Society 158 (2) (2011) A93-A101.

[83] K. Smith, C. Rahn, C. Wang, Control oriented 1D electrochemical model of lithium ion battery, Energy Conversion and Management 48 (2007) 2565-2578.

[84] T.-S. Dao, C. Vyasarayani, J. McPhee, Simplification and order reduction of lithium-ion battery model based on porous-electrode theory, Journal of Power Sources 198 (2012) 329-337.

[85] T.-S. Dao, A. Seaman, J. McPhee, Mathematics-based modeling of a series-hybrid electric vehicle, in: Proc. of the 5th Asian Conference on Multibody Dynamics, 2010

[86] M. Guo, R. White, An approximate solution for solid-phase diffusion on physics-based li-ion cell models, J. Power Sources 198 (2011) 322-328.

[87] X. Hu, S. Stanton, L. Cai, R. White, Model order reduction for solid-phase diffusion in physics-based lithium ion cell models, J. Power Sources 218 (2012) 212-220.

[88] C. Speltino, D. Di Domenico, G. Fiengo, A. Stefanopoulou, Comparison of reduced order lithiumion battery models for control applications, in: Proc. of the joint 48th IEEE Conference on Decision and Control and the 28th Chinese Control Conference, Shanghai, China, 2009, pp. 3276-3281.

[89] V. Subramanian, V. Diwakar, D. Tapriyal, Efficient macro-micro scale coupled modeling of batteries, Journal of the Electrochemical Society 152 (10) (2005) A2002-A2008.

[90] D. Di Domenico, A. Stefanopoulou, G. Fiengo, Reduced order lithium-ion battery electrochemical model and extended kalman filter state of charge estimation, ASME Journal of Dynamic Systems, Measurement and Control-Special Issue on Physical System Modeling. 


\section{Appendix A. Equivalent-Circuit Model Parameters}

Figure C.12 and the following equations show the circuit diagram and circuit parameters used in the equivalent circuit model of Figure C.8. The charge storage circuit is on the top, and the time-response circuit is on the bottom. The current $I(t)$ flowing through the time-response circuit is made to flow in the charge storage circuit. The voltage across the charge storage circuit, $V_{\text {soc }}$, represents the SOC. The state of charge determines the values of the circuit elements in the time-response circuit. The terminals of the time-response circuit represent the terminals of the battery. The parameters used in this model are identical to those used by Chen and Rincón-Mora [24].

$$
\begin{aligned}
C_{\text {cap }} & =3060 \\
V_{\text {oc }} & =-1.031 \exp \left(-35 V_{\text {soc }}\right)+3.685+0.2156 V_{\text {soc }}-0.1178 V_{\text {soc }}^{2}+0.3201 V_{\text {soc }}^{3} \\
R_{\text {series }} & =0.1562 \exp \left(-24.37 V_{\text {soc }}\right)+0.07446 \\
R_{\text {short }} & =0.3208 \exp \left(-29.14 V_{\text {soc }}\right)+0.04669 \\
C_{\text {short }} & =-752.9 \exp \left(-13.51 V_{\text {soc }}\right)+703.6 \\
R_{\text {long }} & =6.603 \exp \left(-155.2 V_{\text {soc }}\right)+0.04984 \\
C_{\text {long }} & =-6056 \exp \left(-27.12 V_{\text {soc }}\right)+4475
\end{aligned}
$$

\section{Appendix B. Nomenclature}

\section{Nomenclature}

$A_{k}$ surface area of electrode $k(k=p, n, s),\left[m^{2}\right]$

$a_{k}$ specific surface area of electrode $k(k=p, n),\left[m^{-1}\right]$

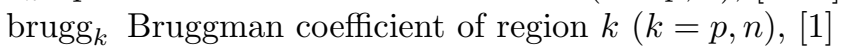

$c_{\mathrm{e}, \text { apprx }}$ assumed solution for electrolyte-phase concentration of $\mathrm{Li}^{+},\left[\mathrm{mol} \cdot \mathrm{m}^{-3}\right]$

$c_{\mathrm{e}, k}$ electrolyte concentration in region $k,\left[\mathrm{~mol} \cdot \mathrm{m}^{-3}\right]$

$c_{\mathrm{e}, k, 0}$ initial electrolyte concentration in region $k,\left[\mathrm{~mol} \cdot \mathrm{m}^{-3}\right]$

$c_{\mathrm{s}, k}$ concentration of $\mathrm{Li}^{+}$ions in the intercalation particle of electrode $k,\left[\mathrm{~mol} \cdot \mathrm{m}^{-3}\right]$

$c_{\mathrm{s}, k, 0}$ initial concentration of $\mathrm{Li}^{+}$ions in the intercalation particle of electrode $k,[\mathrm{~mol}$. $m^{-3}$ ]

$\bar{c}_{\mathrm{s}, k}$ average concentration of $\mathrm{Li}^{+}$ions in the intercalation particle of electrode $k,[\mathrm{~mol}$. $\left.m^{-3}\right]$

$c_{\mathrm{s}, k \text {,surf }}$ concentration of $\mathrm{Li}^{+}$ions on the surface of intercalation particle of electrode $k$, $\left[\mathrm{mol} \cdot \mathrm{m}^{-3}\right]$

$D$ electrolyte diffusion coefficient, $\left[\mathrm{m}^{2} \cdot s\right]$

$D_{\mathrm{s}, k} \mathrm{Li}^{+}$ion diffusion coefficient in the intercalation particle of electrode $k,\left[\mathrm{~m}^{2} \cdot \mathrm{s}\right]$

$F$ Faraday's constant, $\left[C \cdot \mathrm{mol}^{-1}\right]$

$I$ applied current density, $\left[A \cdot m^{-2}\right]$

$J_{k}$ wall-flux of $\mathrm{Li}^{+}$on the intercalation particle of electrode $k,\left[\mathrm{~mol} \cdot \mathrm{m}^{-2} \cdot \mathrm{s}^{-1}\right]$

$K_{k}$ intercalation/deintercalation reaction-rate constant of electrode $k,\left[\mathrm{~mol}^{-0.5} \cdot \mathrm{m}^{2.5}\right.$. $\left.s^{-1}\right]$

$L$ total thickness of cathode-separator-anode, $[m]$

$L_{k}$ thickness of region $k,[m]$ 
$n$ negative electrode

$N$ number of node points for Galerkin's approximation

$p$ positive electrode

$\bar{q}_{\mathrm{s}, k}$ volume-averaged concentration flux of $\mathrm{Li}^{+}$ions in the intercalation particle of electrode $k,\left[\mathrm{~mol} \cdot \mathrm{m}^{-3} \cdot \mathrm{s}^{-1}\right]$

$r$ radial coordinate $[m]$

$R$ universal gas constant

$R_{\mathrm{c}}$ residual function for concentration of $\mathrm{Li}^{+}$in electrolyte-phase

$R_{\mathrm{s}, k}$ radius of intercalation of electrode $k,[\mathrm{~m}]$

$R_{\Phi}$ residual function for electrical potential in electrolyte-phase

$s$ separator

$t_{+} \mathrm{Li}^{+}$transference number in the electrolyte

$T$ absolute temperature, $[K]$

$U_{k}$ open-circuit potential of electrode $k,[V]$

$x$ spatial coordinate, $[m]$

$\alpha$ charge transfer coefficient

$\epsilon_{k}$ volume fraction of region $k$

$\epsilon_{\mathrm{f}, k}$ volume fraction of fillers in region $k$

$\eta$ over-potential, $[V]$

$\eta_{i}$ time-dependent variable of of $i$ 'th basis function for electrolyte-phase concentration, $[s]$

$\theta_{k}$ dimensionless concentration of $\mathrm{Li}^{+}$ions in the intercalation particle of electrode $k$

$\left(\theta_{k}=c_{\mathrm{s}, k} / c_{\mathrm{s}, k, \max }\right)$

$\kappa$ ionic conductivity of electrolyte, $\left[S \cdot m^{-1}\right]$

$\gamma$ activity coefficient

$\kappa_{\text {eff, }, k}$ effective ionic conductivity of the electrolyte in region $k,\left[S \cdot m^{-1}\right]$

$\nu$ stoichiometric coefficient

$\rho_{i}$ time-dependent variable of of $i$ th basis function for electrolyte-phase potential, $[s]$

$\sigma_{k}$ electronic conductivity of solid phase of electrode $k,\left[S \cdot m^{-1}\right]$

$\sigma_{\text {eff }, k}$ effective electronic conductivity of solid phase of electrode $k,\left[S \cdot m^{-1}\right]$

$\Phi$ electrical potential, $[V]$

$\Phi^{0}$ electromotive force at standard conditions, $[V]$

$\Phi_{\mathrm{e}}$ electrolyte-phase potential, $[V]$

$\Phi_{e, \text { apprx }}$ assumed solution for electrolyte-phase potential, $[V]$

$\Phi_{\mathrm{s}}$ solid-phase potential, $[V]$

\section{Appendix C. Captions and Figures}

\section{Appendix C.1. Figure Captions}

1. Simple equivalent series resistance circuit

2. Charge depletion and recovery

3. Dynamic RC battery model

4. Complex equivalent circuit battery model

5. Half-cell EIS circuit

6. A typical electrochemical impedance spectroscopy plot

7. Anatomy of Li-ion cell (colour on Web) 
8. Battery voltage in response to pulsed current. Experimental data from Fig. 5 of [24].

9. Sensitivities for equivalent circuit (top) and electrochemical (bottom) battery models

10. Series HEV block diagram

11. Battery voltage and SOC over EPA drive cycle

12. Battery circuit

Appendix C.2. Figures

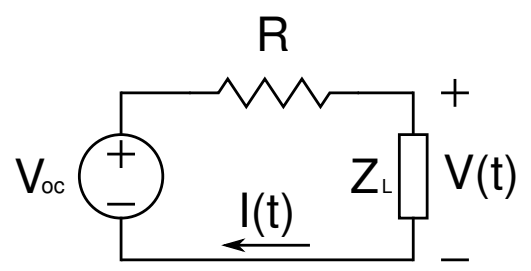

Figure C.1:

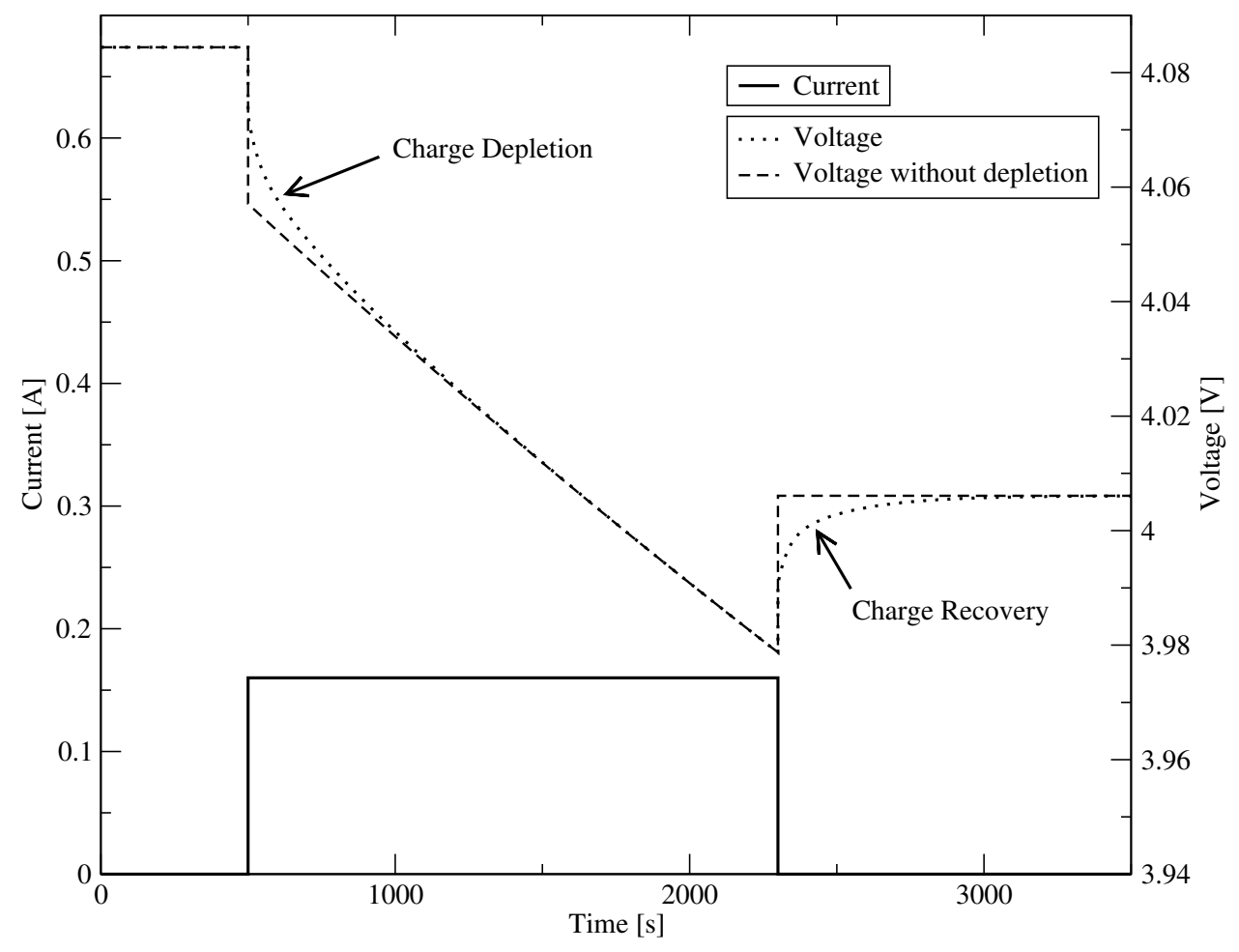

Figure C.2: 


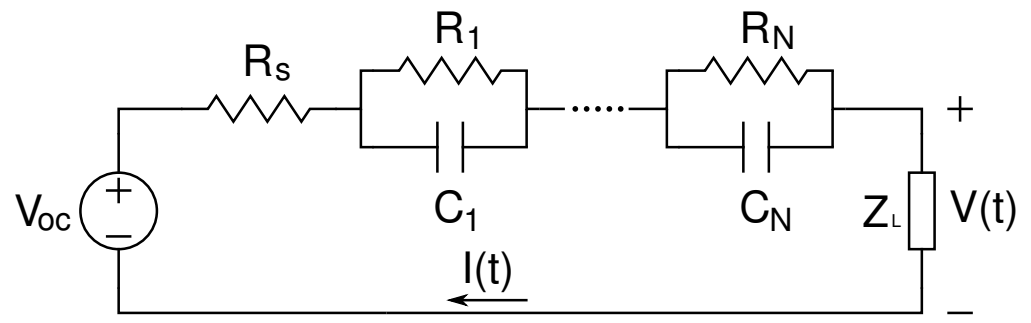

Figure C.3:

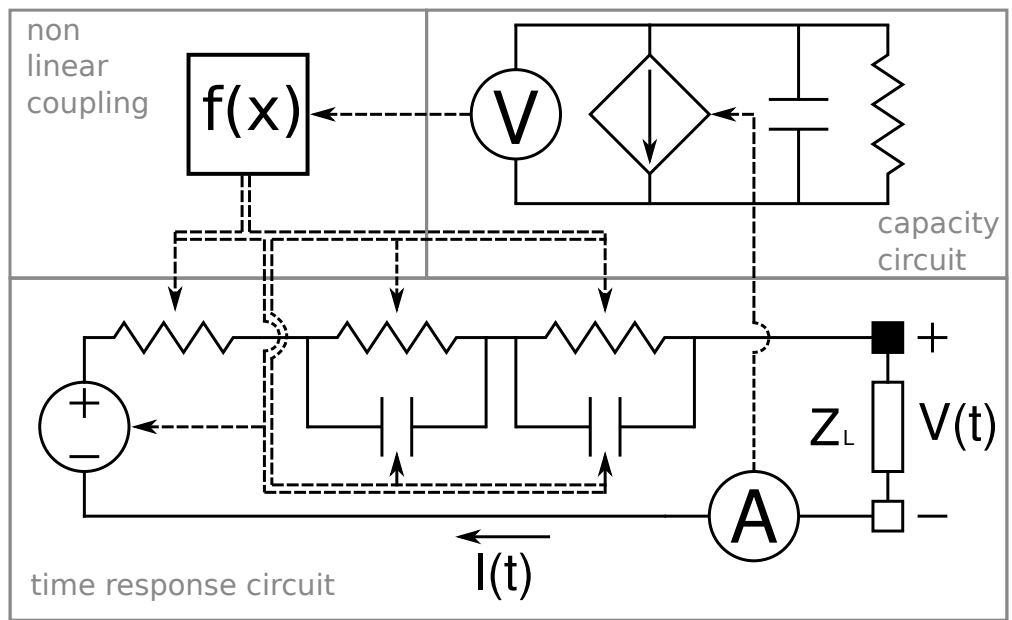

Figure C.4:

Appendix D. Tables 


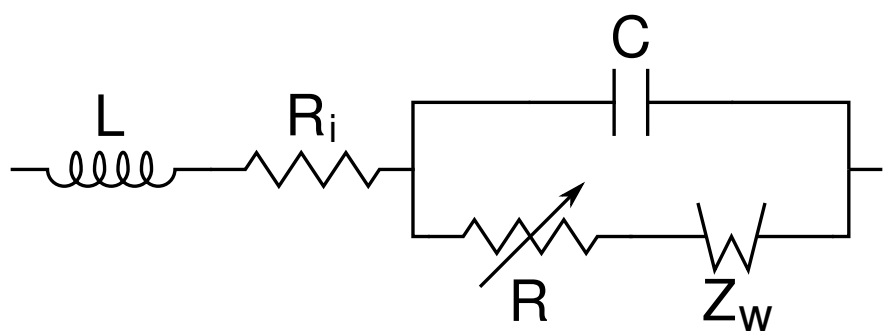

Figure C.5:

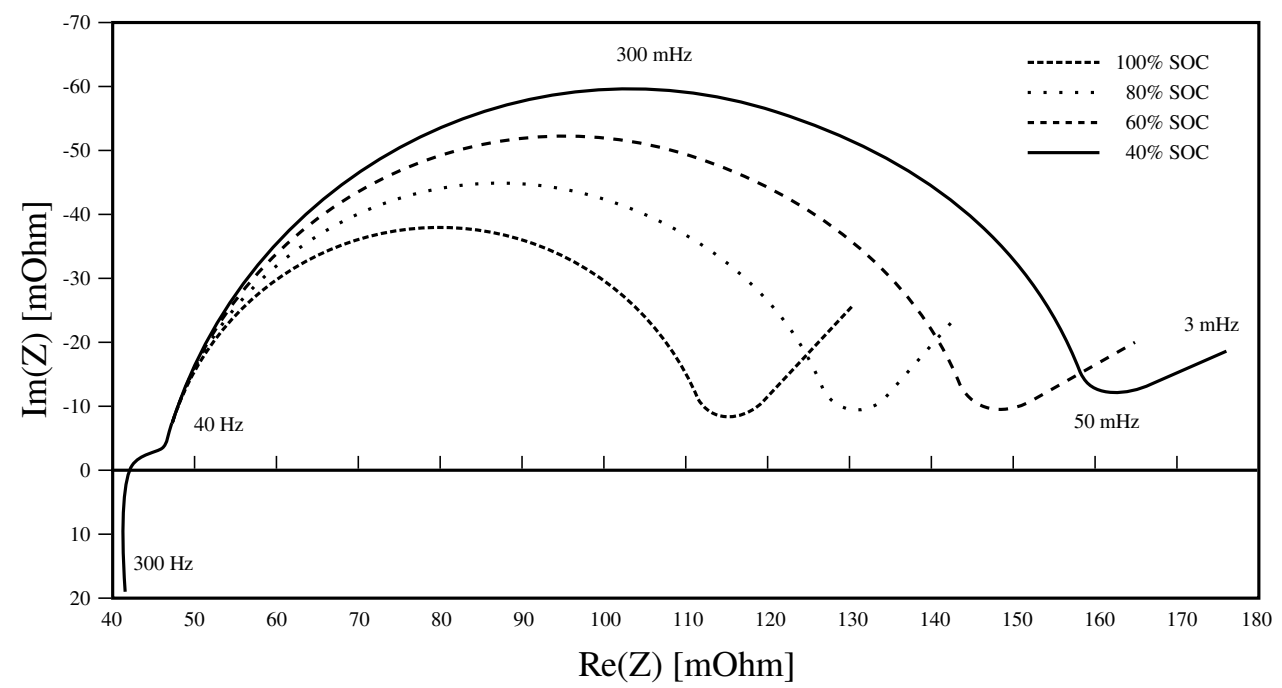

Figure C.6: 


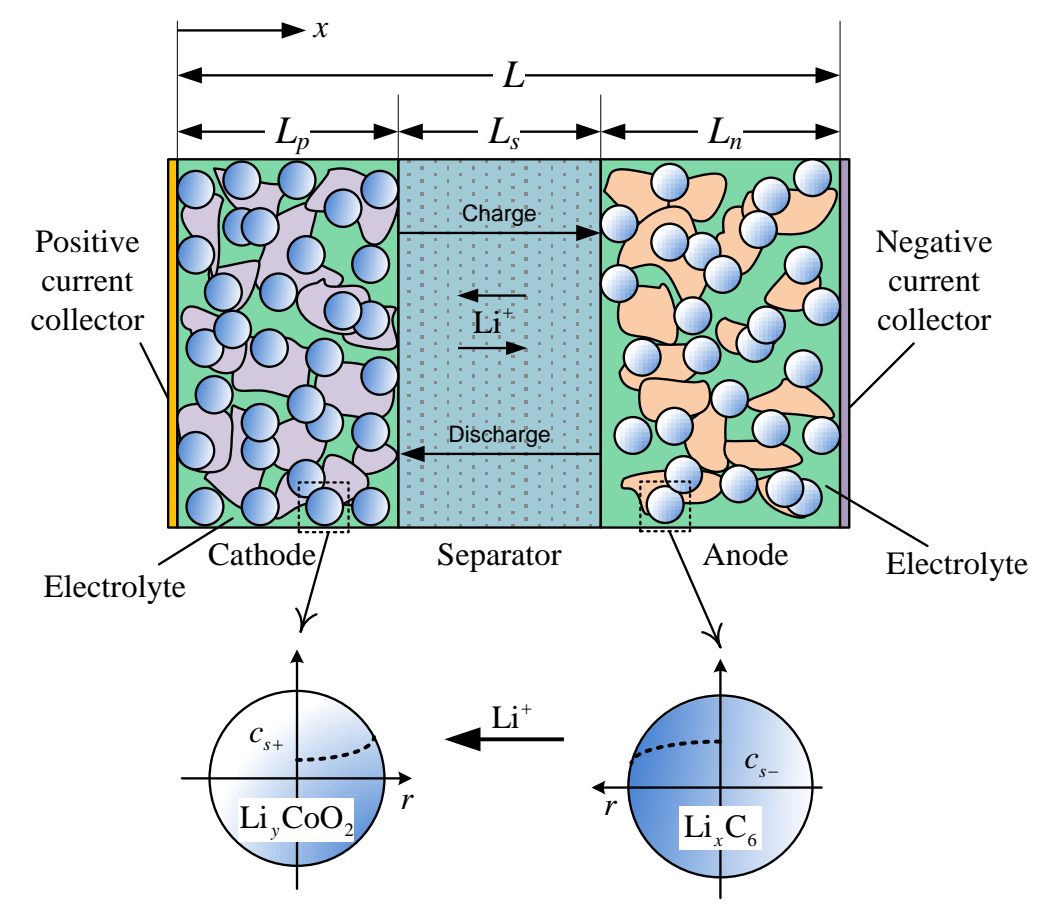

Figure C.7:

Table D.1: Battery parameters.

\begin{tabular}{lllll}
\multicolumn{5}{c}{ Table D.1: Battery parameters. } \\
\hline Symbol & Unit & $\begin{array}{l}\text { Positive } \\
\text { electrode }\end{array}$ & Separator & $\begin{array}{l}\text { Negative } \\
\text { electrode }\end{array}$ \\
\hline$\sigma_{k}$ & $\mathrm{~S} \mathrm{~m}^{-1}$ & 100 & & 100 \\
$\epsilon_{\mathrm{f}, k}$ & & 0.025 & 0.0326 \\
$\epsilon_{k}$ & & 0.385 & 0.724 & 0.485 \\
brugg & & 1.5 & 1.5 & 1.5 \\
$D_{\mathrm{s}, k}$ & $\mathrm{~m}^{2} \mathrm{~s}^{-1}$ & $1 \times 10^{-14}$ & & $3.9 \times 10^{-14}$ \\
$D$ & $\mathrm{~m}^{2} \mathrm{~s}^{-1}$ & $7.5 \times 10^{-11}$ & $7.5 \times 10^{-11}$ & $7.5 \times 10^{-11}$ \\
$K_{k}$ & $\mathrm{~mol}^{-0.5} \mathrm{~m}^{2.5} \mathrm{~s}^{-1}$ & $2.344 \times 10^{-11}$ & & $5.0307 \times 10^{-11}$ \\
$c_{\mathrm{s}, k, \mathrm{max}}$ & $\mathrm{mol} \mathrm{m}^{-3}$ & 40554 & & 30555 \\
$c_{\mathrm{S}, k, 0}$ & $\mathrm{~mol} \mathrm{~m}^{-3}$ & $0.4955 \times 40554$ & & $0.8551 \times 30555$ \\
$c_{\mathrm{e}, 0}$ & $\mathrm{~mol} \mathrm{~m}^{-3}$ & 5000 & 5000 & 5000 \\
$R_{\mathrm{s}, k}$ & $\mathrm{~m}_{L_{k}}$ & $2.0 \times 10^{-6}$ & & $2.0 \times 10^{-6}$ \\
$R_{\mathrm{SEI}}$ & $\Omega \mathrm{m} \mathrm{m}^{2}$ & $133 \times 10^{-6}$ & $20 \times 10^{-6}$ & $139 \times 10^{-6}$ \\
$t_{+}$ & & & & $5 \times 10^{-3}$ \\
$F$ & $\mathrm{C} \mathrm{mol}^{-1}$ & 0.363 & 0.363 & 0.363 \\
$R$ & $\mathrm{~J} \mathrm{~mol}^{-1} \mathrm{~K}^{-1}$ & & 96487 & \\
\hline
\end{tabular}




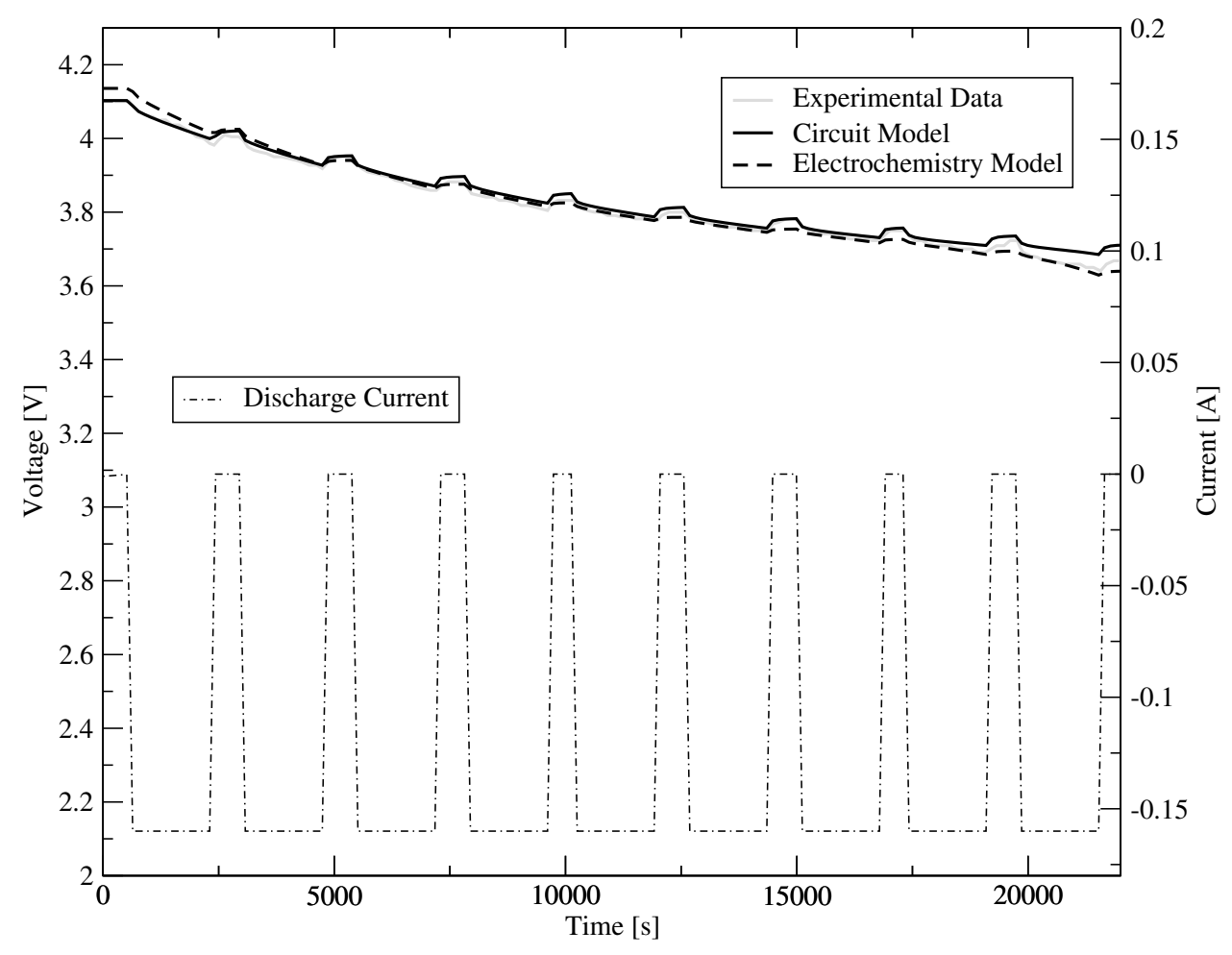

Figure C.8:

Table D.2: Electrochemical sensitivity symbols and names

\begin{tabular}{llllll}
\hline & Parameter & \multicolumn{3}{c}{ Parameter } & Parameter \\
Symbol & Name & Symbol & Name & Symbol & Name \\
\hline$A_{n}$ & An & $\epsilon_{\mathrm{f}, n}$ & efn & $L_{n}$ & Ln \\
$A_{p}$ & Ap & $\epsilon_{\mathrm{f}, p}$ & efp & $L_{p}$ & Lp \\
$A_{s}$ & As & $\epsilon_{n}$ & en & $L_{s}$ & Ls \\
$c_{\mathrm{e}, 0}$ & ce0 & $\epsilon_{p}$ & ep & $R_{\mathrm{s}, n}$ & Rsn \\
$c_{\mathrm{s}, n, \max }$ & csnmax & $\epsilon_{s}$ & es & $R_{\mathrm{s}, p}$ & Rsp \\
$c_{\mathrm{s}, p, \max }$ & cspmax & & & & \\
\hline
\end{tabular}




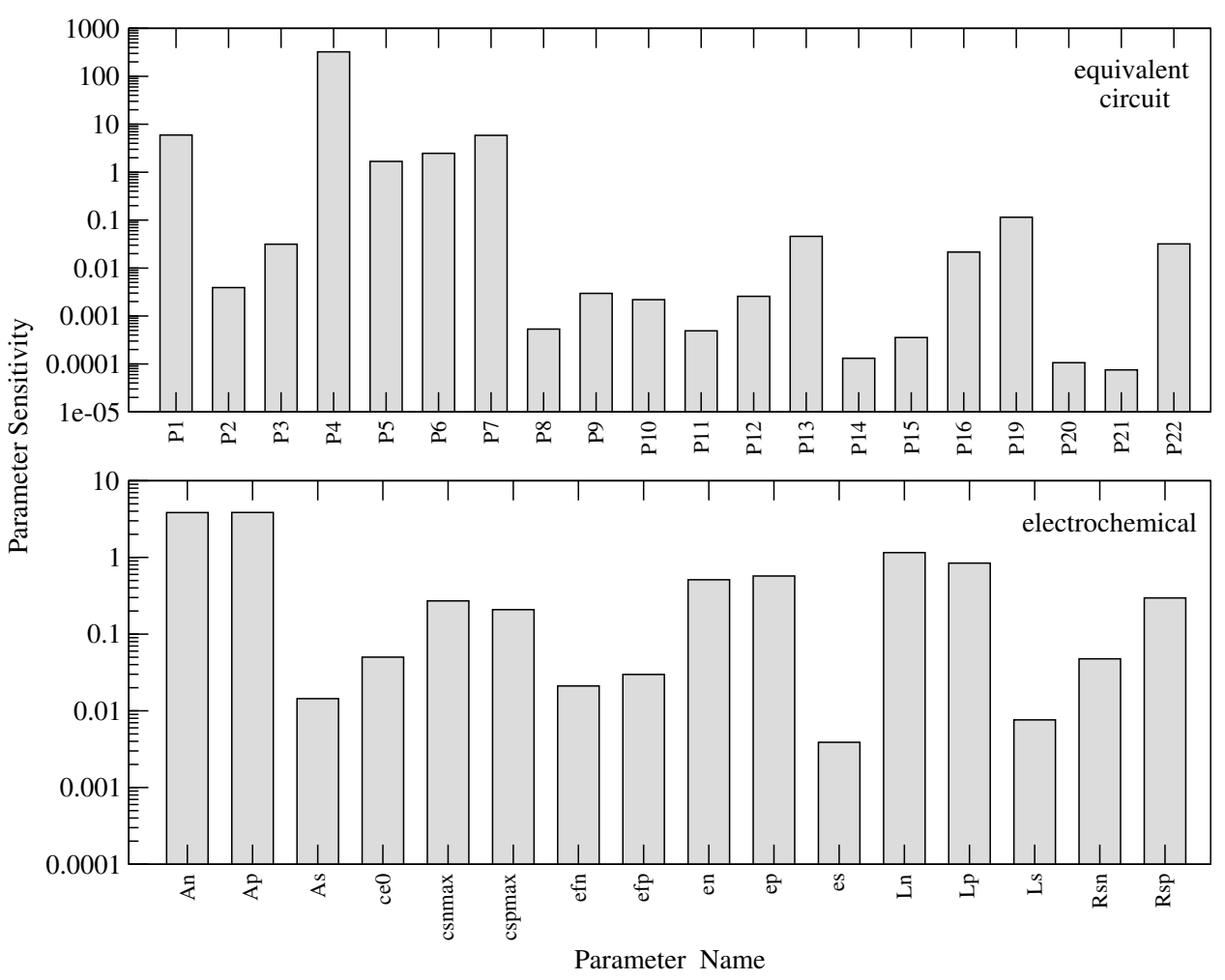

Figure C.9:

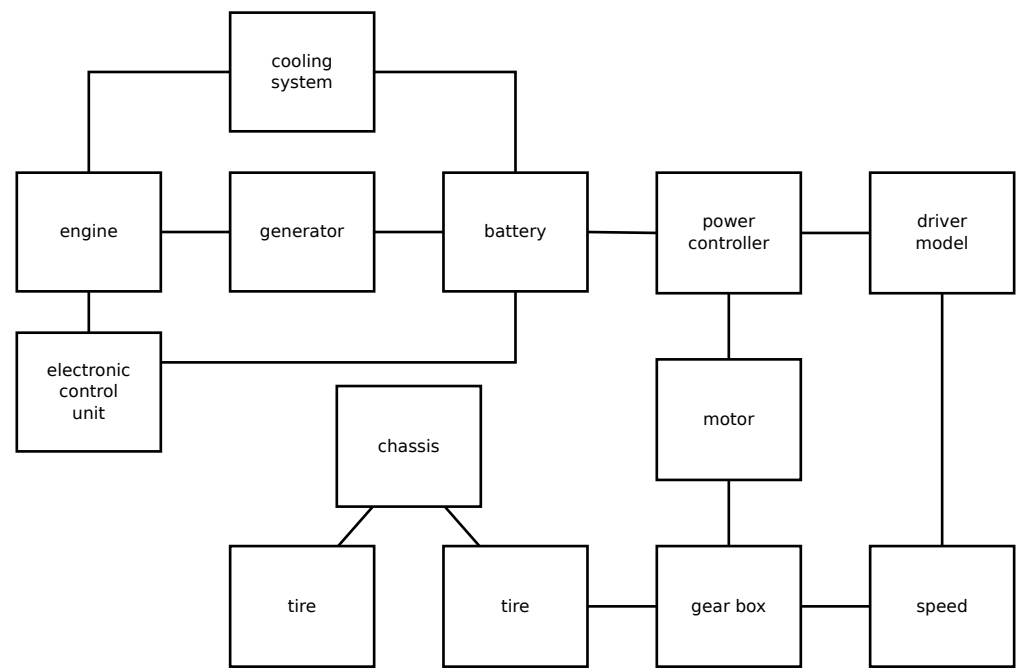

Figure C.10: 


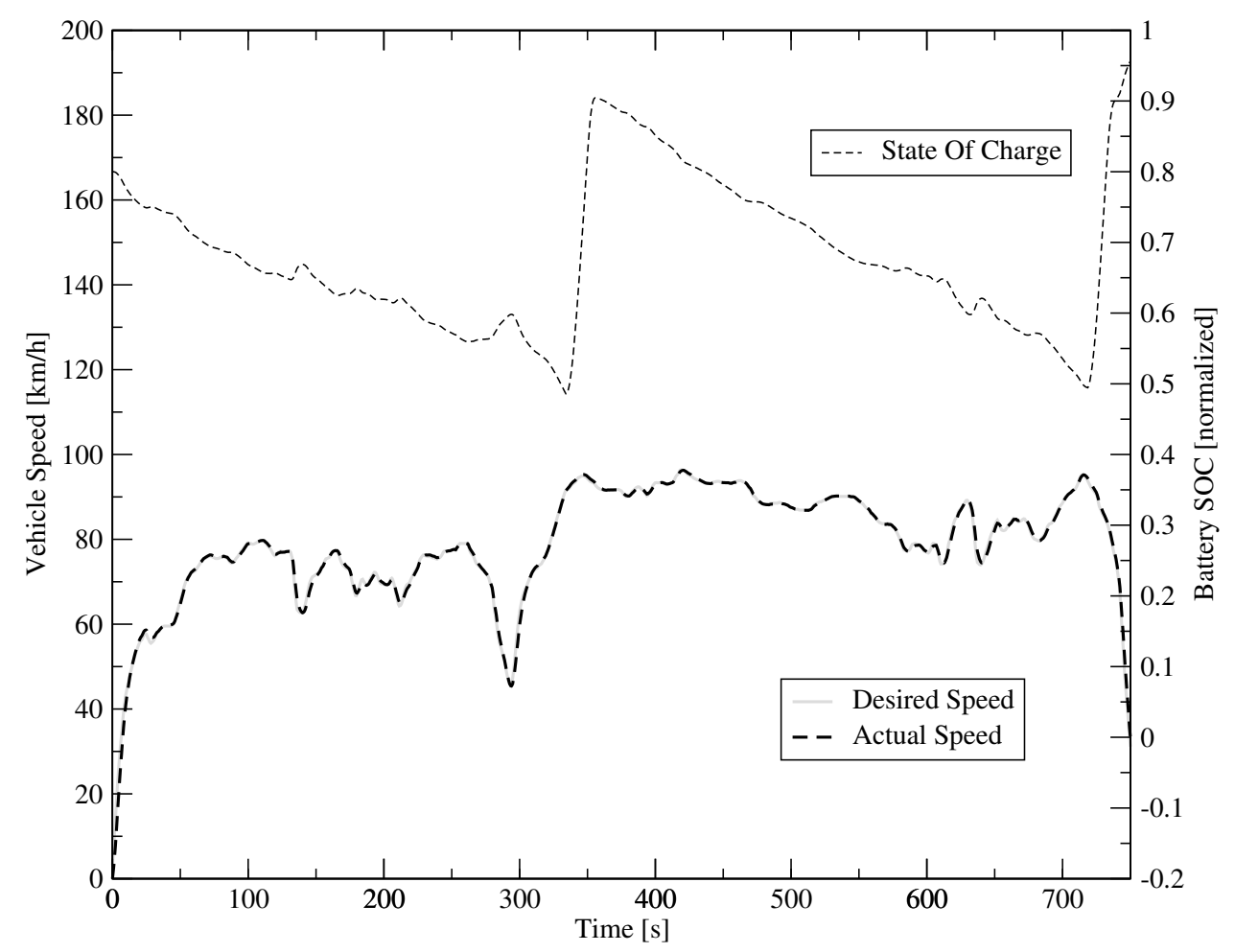

Figure C.11:

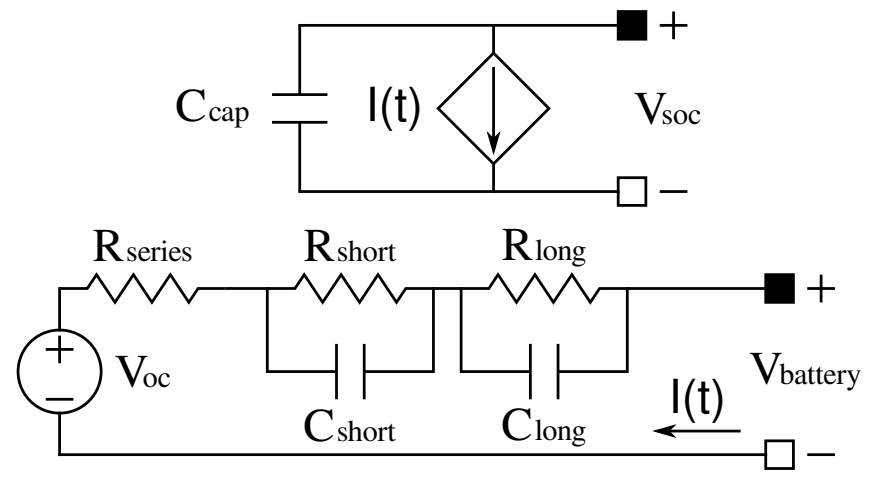

Figure C.12: 